\title{
RELACIONAMENTO INTERPESSOAL DA EQUIPE DE ENFERMAGEM X ESTRESSE: LIMITAÇÕES PARA A PRÁTICA*
}

Cláudia Cristiane Filgueira Martins', Viviane Euzébia Pereira Santos², Marta Silvânere Pereira³, Natally Pereira dos Santos ${ }^{3}$

${ }^{1}$ Enfermeira. Mestre em Enfermagem. Universidade Federal do Rio Grande do Norte. Natal-RN-Brasil. 2Enfermeira. Doutora em Enfermagem. Universidade Federal do Rio Grande do Norte. Natal-RN-Brasil. ${ }^{3}$ Acadêmica. Universidade Federal do Rio Grande do Norte. Natal-RN-Brasil.

RESUMO: Estudo descritivo com o objetivo de analisar a relação entre o relacionamento interpessoal e o estresse dos profissionais da equipe de enfermagem da unidade de terapia intensiva de um hospital universitário de Natal-RN, Brasil. Os dados foram coletados através de entrevista semiestruturada entre setembro e outubro de 2011, com 21 integrantes da equipe de enfermagem. Os dados foram tratados a luz da análise temática de conteúdo. Essa análise permitiu a elaboração de duas categorias: Relacionamento interpessoal da equipe de enfermagem na unidade de terapia intensiva: reflexos na prática dos profissionais; e Comunicação deficitária entre os membros da equipe. Foi possível evidenciar o contexto de relações frágeis que existe dentro da equipe de enfermagem em decorrência de sucessivos momentos de estresse vivenciados em sua dinâmica laboral. Assim, faz-se necessário que esses profissionais se sensibilizem e percebam a importância de cuidar de si em seu ambiente de trabalho.

DESCRITORES: Enfermagem; Estresse psicológico; Relações interpessoais.

\section{THE NURSING TEAM'S INTERPERSONAL RELATIONSHIPS VERSUS STRESS: LIMITATIONS FOR PRACTICE}

\begin{abstract}
This descriptive study aimed to analyze the relations between the interpersonal relationships and stress of the professionals of the nursing team of the intensive care unit in a teaching hospital in Natal, in the State of Rio Grande do Norte (RN), Brazil. The data were collected through semi-structured interviews held between September and October 2011 with 21 members of the nursing team. The data were treated using thematic content analysis. This analysis allowed the elaboration of two categories: The nursing team's interpersonal relationships in the intensive care unit: reflections in the professionals' practice; and Inadequate communication between the team members. It was possible to evidence the context of fragile relationships which exist within the nursing team as a result of successive times of stress experienced in their work dynamic. Thus, it is necessary for these professionals to raise their awareness and notice the importance of caring for themselves in their work environment.
\end{abstract}

DESCRIPTORS: Nursing; Psychological stress; Interpersonal relationships.

\section{RELACIÓN INTERPERSONAL DEL EQUIPO DE ENFERMERÍA X ESTRÉS: LIMITACIONES PARA LA PRÁCTICA}

RESUMEN: Estudio descriptivo cuya finalidad fue analizar las relaciones interpersonales en el ambiente del estrés de los profesionales del equipo de enfermería de la unidad de terapia intensiva de un hospital universitario de Natal, RN, Brasil. Los datos fueron obtenidos a través de entrevista semiestructurada entre septiembre y octubre de 2011, con 21 integrantes del equipo de enfermería. Los datos fueron trabajados a la luz del análisis temático de contenido. Ese análisis permitió la elaboración de dos categorías: Relaciones interpersonales del equipo de enfermería en la unidad de terapia intensiva: reflejos en la práctica de los profesionales; y Falta de comunicación entre los miembros del equipo. Fue posible evidenciar el contexto de relaciones débiles que hay en el equipo de enfermería en razón de sucesivos momentos de estrés de su dinámica laboral. Así, es necesario que eses profesionales se sensibilizen y perciban la importancia de cuidar de si en su ambiente de trabajo.

DESCRIPTORES: Enfermería; Estrés psicológico; Relaciones interpersonales.

* Recorte da dissertação "No caleidoscópio o estresse da unidade de terapia intensiva de um hospital universitário em Natal-RN", apresentada ao Programa de Pós-graduação em Enfermagem da Universidade Federal do Rio Grande do Norte.

Recebido: $26 / 11 / 2013$ Finalizado: 31/03/2014

\section{Autor Correspondente:}

Cláudia Cristiane Filgueira Martins

Universidade Federal do Rio Grande do Norte.

Rua Sebastião Barreto, 91 - 59080-480 - Natal-RN-Brasil

E-mail: claudiacrisfm@yahoo.com.br 


\section{INTRODUÇÃO}

As atuais transformações do mundo do trabalho acarretam consequências diretas na vida e saúde dos trabalhadores, de forma incisiva e, em sua maioria, negativamente. $\mathrm{O}$ aumento do ritmo de trabalho implica em consumo de energia física e psicológica desses levando-os a desenvolver um quadro de estresse em sua vida pessoal e profissional.

Assim, ocorre uma desarmonia na relação homem-trabalho, possibilitando inúmeras alterações na saúde em decorrência do ritmo e do próprio processo de trabalho. Na área da saúde, isso pode ser evidenciado sob vários aspectos, dentre eles destaca-se: trabalhadores cansados excessivamente devido às longas jornadas, desencadeando problemas físicos e psíquicos, respondendo na forma de doenças crônicas, como a hipertensão arterial sistêmica e diversas consequências osteomusculares, dentre essas cabe destacar o estresse ocupacional ${ }^{(1)}$.

O estresse é definido como uma resposta fisiológica, psicológica e comportamental de um indivíduo buscando adaptar-se às exigências do ambiente e às internas, sendo visto como estimulante e fonte de equilíbrio( ${ }^{(2)}$. Os malefícios só se desenvolvem quando esse estado de adaptação é ineficaz, podendo resultar em um processo patológico. O estresse ocupacional é considerado como uma consequência do desequilíbrio entre as exigências do trabalho, a capacidade de enfrentamento e necessidades do trabalhador ${ }^{(3)}$. Dentre os principais fatores precursores encontram-se os aspectos da organização, administração e qualidade das relações humanas ${ }^{(4)}$.

Na profissão de enfermagem esse contexto ganha destaque, visto que os regimes de plantões, a dupla e até tripla jornada de atuação, bem como as condições de trabalho favorecem o aparecimento de consequências negativas no corpo e na mente desses trabalhadores, bem como à assistência prestada ${ }^{(5)}$. Assim, essa dinâmica laboral intensa influencia, diretamente, as relações entre esses profissionais, bem como entre esses e os demais membros da equipe de saúde, o que pode levar às relações frágeis, carentes de vínculos e uma equipe fragmentada, desse modo, gera assistência mecanizada ao paciente. Além disso, essas relações podem ser afetadas devido às circunstâncias do cotidiano profissional e agravadas pelo ambiente de trabalho em que atuam; trazendo confrontos diversos, com situações estressantes vivenciadas dentro do ambiente de trabalho.

Nesse contexto, têm-se como objeto de estudo as relações interpessoais e sua interface com estresse na unidade de terapia intensiva (UTI). Em que, foi elaborada como questão norteadora da pesquisa: como o estresse do profissional de enfermagem influencia o relacionamento interpessoal desses trabalhadores em seu ambiente de trabalho?

Dessa forma, esse estudo teve como objetivo analisar a relação entre o estresse do profissional e relacionamento interpessoal dos trabalhadores que compõe a equipe de enfermagem da UTI de um hospital universitário na cidade de Natal-RN, Brasil.

Cabe destacar que este estudo se fez relevante devido a grande influencia que o estresse acarreta no cotidiano e nas ações de diversos profissionais de saúde, em especial os da enfermagem. Deste modo, é desejável demonstrar como esse fator negativo relacionado ao trabalho é capaz de desencadear desordens diversas ao serviço, na forma de comunicações e relações frágeis entre os membros das equipes, e afetando diretamente o cuidado destinado aos pacientes por eles assistidos.

\section{MÉTODO}

Trata-se de um estudo qualitativo, de abordagem descritiva, realizada junto à equipe de enfermagem da UTI de um hospital universitário em Natal-RN, Brasil.

A escolha do campo se deu pelo fato de ser um setor de referência para o Estado do Rio Grande do Norte, bem como existir um grande fluxo de estudantes e estagiários de diversas áreas, podendo haver relações interpessoais, por vezes, conflituosas. Além disso, a grande quantidade de profissionais da saúde em um único ambiente hospitalar prestando assistência a pacientes graves em estado terminal pode influenciar no modo como se atua nesse ambiente.

O cenário estudado tem capacidade de 12 leitos, sendo 8 para UTI geral e 4 para neurocardiologia. Todos são leitos do Sistema 
Único de Saúde (SUS) e, em sua maioria, os pacientes possuem patologias crônicas ou estão em tratamento pós-operatório. O fato de ser um ambiente universitário acarreta em um grande fluxo de alunos das mais diferentes áreas de graduação e pós-graduação (residências) em saúde, bem como, um grande contingente de colaboradores por turno.

Desse modo, a escolha da abordagem proporcionou uma maior aproximação entre os pesquisadores e os sujeitos estudados, visando compreender a realidade estudada. Possibilitando, ainda, cruzamento e integração entre a literatura e a questão do estudo, conduzindoà abordagem integral e fidedigna entre as percepções dos integrantes de equipes acerca da realidade que vivenciam ${ }^{(6)}$.

A coleta de dados ocorreu no período de setembro e outubro de 2011. Para esta etapa da pesquisa os participantes foram selecionados em conformidade com os seguintes critérios de inclusão: ter disponibilidade para responder a entrevista semiestruturada; atuar há mais de seis meses no referido setor e pertencer ao quadro efetivo de funcionários. Foram excluídos aqueles profissionais que estavam em licença por qualquer motivo.

O estudo foi aprovado no Comitê de Ética em Pesquisa-HUOL, comn ${ }^{\circ}$ 0037.0.294.000-11 enorteado a partir das recomendações éticas estabelecidas nas diretrizes para pesquisas com seres humanos ${ }^{(7)}$.

Foi utilizado um roteiro de entrevista semiestruturada com questões acerca do estresse e a rotina diária desses profissionais no ambiente de trabalho e o relacionamento interpessoal entre os membros da equipe. Participaram do estudo 21 integrantes da equipe de enfermagem, sendo 5 enfermeiros e 16 técnicos de enfermagem. Os entrevistados foram denominados por ordem cronológica de período de realização das entrevistas e identificados por Enfermeiro (E1, E2...) e Técnico de Enfermagem (TE1, TE2...).

Esses dados foram transcritos e analisados segundo o método de análise de conteúdo temática, permitindo uma avaliação sistemática de mensagens dos interlocutores e síntese dos dados conforme as seguintes etapas: pré-análise, análise e interpretação(8). Essas fases distintas permitiram a organização de ideias, a codificação das informações e ao se alcançar as unidades de significação das entrevistas foram definidas as temáticas que fundamentaram o estudo.

\section{RESULTADOS}

Com a tabulação e interpretação desses dados, foi possível perceber que $80 \%$ dos profissionais são do sexo feminino,; estão na faixa etária de 30 a 39 anos (52,3\%); são casadas (52,3\%) e possuem duplo vínculo (65,7\%).

Após sucessivas leituras e análise com a literatura investigada emergiram duas categorias sendo estas intituladas de Relacionamento interpessoal da equipe de enfermagem na UTI: reflexos na prática dos profissionaise, Comunicação deficitária entre os membros da equipe.

Os recortes abaixo esboçam as falas da primeira categoria: Relacionamento interpessoal da equipe de enfermagem na UTI: reflexos na prática dos profissionais. Os entrevistados deste estudo caracterizaram momentos estressantes do trabalho em equipe o relacionamento interpessoal. Isso pode ser visto nos recortes a seguir:

Alguns fatores que a gente tá tendo em termo de convívio entre os próprios enfermeiros, por causa de escala, alguns problemas da própria equipe, falando exclusivamente dos enfermeiros, então a gente já recebe o plantão de uma forma que a gente já fica meio chateado, ou pensando que a gente tá fazendo alguma coisa de errado, em termos de relacionamento interpessoal com os enfermeiros, aí você já trabalha tenso. (E1)

Eu acho que às vezes estressa é que acontece assim, picuinhas [...] como tem em qualquer emprego [...] é cobrança desnecessária, que a gente sabe que tá trabalhando direito. (TE5)

A gente tem uma equipe muito grande, é um hospital universitário e a gente tem constantemente entrada e saída de aluno de várias profissões, um número de pessoas muito grande circulando na UTI, me incomoda, pra mim é um fator muito estressante. (E4)

$\mathrm{Na}$ realidade investigada, foram percebidos como influenciadores do relacionamento interpessoal e potencializadores do estresse e tensão no ambiente de trabalho: problemas com a escala de turno, falta de interação entre a equipe, carências na resolução dos problemas típicos 
do setor, cobranças desnecessárias, excesso de alunos de diferentes categorias no setor por se tratar de um hospital universitário.

Além disso, o profissional de enfermagem atua em ritmos intensos, longas jornadas e de forma mecanizada, contribuindo para um ambiente de atuação repleto de tensões, as quais podem ser refletidas no corpo, no comportamento e na mente do indivíduo, ocasionando problemas ao seu local de trabalho.

Os entrevistados referiram, também, que o fato de conviver em um ambiente que propicia situações de estresse contribui para aumentar o nível de tensão vivenciado nesse ambiente e relataram os sentimentos que permeiam o relacionamento interpessoal da equipe, que têm como base a angústia e a tensão no ambiente de atuação profissional. O convívio com situações negativas, podem ser entendidas e vivenciadas como estressores principais da equipe atuante na terapia intensiva.

Com relação a segunda categoria do estudo: Comunicação deficitária entre os membros da equipe, a equipe mencionou que há presença de uma ruptura neste processo, como referenciada nos recortes:

Quem trabalha em setor fechado sabe que, se a equipe não interagir, não fica tudo bem [...] Às vezes falta alguma coisa, não faz uma observação que foi passada a seu paciente, aí, assim, gera um bloqueio no plantão. (TE2)

A passagem de plantão me estressa um pouco, porque é feita em grupo, tem que ouvir todos os outros pacientes, aí você não entende o que seu paciente tem, e ainda tem que passar para os outros. (TE6)

O que me estressa é essa rotatividade de pessoal [...] aquela, aquela multidão de gente que fica por aqui, mas por sorte é que é até seis horas por aqui. (TE7)

Os profissionais em questão descrevem como fatores influenciadores no déficit de comunicação entre os membros da equipe: a passagem de plantão, realizada em grupo, na qual o número de casos discutidos juntos os impede de compreender as necessidades reais dos pacientes ao quais foram designados; e a rotatividade de pessoal, pois por se tratar de um hospital-escola há sempre um grande número de discentes, mas os mesmos não permanecem no setor, impedindo que haja uma continuidade da relação.

Além disso, reconhecem a importância da comunicação no ambiente da UTI, uma vez que caso não haja interação adequada entre os profissionais, há uma ruptura no processo de cuidado ao paciente, impedindo que o plantão flua de maneira satisfatória.

\section{DISCUSSÃO}

\section{Relacionamento interpessoal da equipe de enfermagem na UTI: reflexos na prática dos profissionais}

O relacionamento interpessoal é um fator determinante para o desenvolvimento do cuidado destinado aos pacientes em terapia intensiva. Afinal, esse ambiente, onde as relações profissionais são mais intensas e os profissionais tornam-se mais próximos uns dos outros, acaba sendo palco de relações conflituosas.

Faz-se necessário compreender que, em um ambiente em que a interação com a equipe é diária, haja um equilíbrio harmonioso entre esses sujeitos. Uma vez que esses relacionamentos influenciam no cotidiano e podem dificultar o desenvolvimento e a realização das atividades na equipe ${ }^{(9)}$.

O relacionamento interpessoal pode influenciar positivamente o cotidiano de trabalho da equipe, através de relações harmoniosas que propiciem o aprimoramento das pessoas, ou negativamente, por meio de relações desfavoráveis, tensas, dificultando o desenvolvimento e a realização das atividades ${ }^{(10)}$.

Essas influências negativas na equipe podem ser visualizadas se confrontarmos características inerentes ao trabalho de enfermagem. A primeira delas se remete ao fato de ser uma profissão que envolve o cuidado em todas suas interfaces, fazendo com que o profissional interaja com pessoas doentes em todas as fases da vida, vivenciando perdas, sofrimento e morte no seu ambiente de atuação, o que pode gerar uma 
sobrecarga expondo esse profissional ao desgaste emocional ${ }^{(11)}$.

Ao vivenciarem essas situações estressantes, o relacionamento interpessoal entre os profissionais pode ficar comprometido, em virtude da elevada carga tensional a qual o profissional está submetido. Apesar disso, é imprescindível a manutenção de convivência saudável com a equipe a fim de que o cuidado terapêutico adequado ao paciente não seja prejudicado ${ }^{(9)}$.

De acordo com estudo realizado num hospital do interior do Rio Grande do Sul- Brasil, que objetivou conhecer as relações interpessoais da equipe de enfermagem, podem ser citados outros fatores que influenciam o relacionamento interpessoal, como o número de pessoas por turno de trabalho, o grau de resolubilidade que cada pessoa é capaz de oferecer aos problemas enfrentados, o poder da chefia, grau de flexibilidade, de cobranças, bem como a satisfação ou não em realizar determinada tarefa ${ }^{(9)}$.

É o contato direto com uma rotina desse tipo que favorece a aproximação ou o desprendimento com relação aos demais profissionais da equipe. Sendo assim, é esse convívio que é capaz de gerar uma série de consequências à atuação e ao cuidado prestado por esses profissionais. Como exemplo pode-se citar: a instabilidade de relações, o que torna o ambiente de trabalho negativo; as emoções que determinam como conduzir os conflitos no grupo; e o cuidado destinado ao paciente também é prejudicado, pois a assistência acaba sendo permeada por fatores negativos.

Faz-se necessário compreender que a equipe de enfermagem estabelece entre si e os demais membros envolvidos uma teia de relacionamentos grupais de caráter complexo e que exige um gerenciamento adequado dessas relações por parte de todos os membros da equipe.

Participar de uma equipe é compreender que as relações transcendem o vínculo pessoal, afinal existem outros, fora dessa, que necessitam de uma boa interação. $\mathrm{O}$ trabalho multiprofissional é uma ferramenta imprescindível ao lidar com pacientes e familiares devido a complexidade inerente aos cuidados de saúde. É preciso suscitar uma reflexão acerca da diversidade individual e articular os membros da equipe para perceber e focalizar desvios na convivência e elaborar meios eficientes de alívio das tensões para que consequências negativas não se sobressaiam em relação às positivas ${ }^{(12)}$.

\section{Comunicação deficitária entre os membros da equipe}

Outra categoria que emergiu do relacionamento interpessoal foi a comunicação deficitária entre os membros da equipe de trabalho. Sabe-se que esta se constitui em um importante instrumento para o alcance das metas do cuidado. Além disso, é através dela que o profissional pode exprimir suas ações, opiniões, medos e atitudes dentro do ambiente em que trabalha.

Em um ambiente de trabalho como o hospitalar, a comunicação seja verbal ou não-verbal faz parte do processo de cuidar em enfermagem, uma vez que potencializa a interação entre a equipe, os pacientes e familiares, por isso precisa ser conduzida com ética e zelo a fim de tornar as relações humanas mais harmônicas ${ }^{(10)}$.

No entanto, na terapia intensiva esse ato tornase um desafio, principalmente entre os membros da equipe. Isso ocorre pelo fato de que esse ambiente possui um aparato tecnológico intenso e complexas situações que envolvem os profissionais em uma rotina que exige um cuidado contínuo.

Caso esse processo comunicativo seja ineficiente entre os membros da equipe, ocorrerá o surgimento de fatores geradores de insatisfação nas instituições de saúde, podendo acarretar uma má qualidade da assistência prestada. Acredita-se que o enfermeiro por está em contato constantemente com a equipe multiprofissional deve ser o elo da cadeia comunicativa ${ }^{(13-15)}$. O fato de ter que contar e interagir com os outros membros é de fundamental importância para que ocorra uma continuidade do cuidado que é destinado ao paciente. Isso traduz o significado do trabalho em equipe, ou seja, sua dinamicidade.

Assim pode-se dizer que o papel do enfermeiro não se restringe ao desenvolvimento de habilidades técnicas, mas é preciso também uma ação cuidativa abrangente, o que implica desenvolver a capacidade de comunicação ${ }^{(14)}$. Através do processo comunicativo eficiente é que se pode esperar pela formação do vínculo, respeito, afeto, confiança, indispensáveis para uma boa relação no ambiente de trabalho e 
consequentemente para a continuidade do processo de cuidado.

A interrupção do processo comunicativo pôde ser percebida no momento da passagem de plantão, que é o exercício da comunicação pelos profissionais de enfermagem e ocasiona a interrupção do cuidado se esse processo não for realizado adequadamente, sendo considerada, inclusive, fonte de estresse para alguns profissionais.

Na rotina de trabalho, na UTI investigada, cada técnico de enfermagem possui uma atribuição com um paciente, e no momento da passagem do plantão tem que entender o que se passa com todos os pacientes em um grande grupo. É conhecido que a a passagem de plantão é um momento que a equipe tem para assegurar a continuidade da assistência prestada, relatando eventuais pendências, estado de saúde dos pacientes, burocracias típicas do setor, e é realizada por meio exclusivamente do processo comunicativo.

Ao analisar o processo de comunicação estabelecida na passagem de plantão, alguns autores $^{(13-15)}$ evidenciam pontos negativos, como divergência de opiniões entre os membros da equipe, presença de ruídos que provocam a desconcentração, desatenção e falhas de compreensão da comunicação.

No caso dessa UTI, por ser um hospital escola, a quase todo momento há o acúmulo de pessoas nas unidades, o que provoca um acúmulo de ruídos na comunicação, podendo gerar uma incompreensão da passagem de plantão, por exemplo. No entanto, cabe ressaltar que deve haver uma percepção dessas falhas que estão estressando os profissionais e comprometendo o processo comunicativo da referida equipe, afim de que o cuidado destinado aos pacientes não seja afetado.

\section{CONSIDERAÇÕES FINAIS}

O presente estudo possibilitou a análise e a reflexão acerca do estresse dos profissionais de enfermagem atuantes em UTI e sua relação com o relacionamento interpessoal da equipe. Dentre os elementos potencializadores do estresse e que influenciam diretamente o relacionamento interpessoal dessa equipe, foram citados: problemas advindos da escala de turnos; falta de interação entre os membros da equipe; a carência de resolubilidade na resolução de problemas do setor; cobranças desnecessárias; excesso de alunos no setor e a comunicação deficitária.

Tais estressores trazem consequências diretas ao relacionamento interpessoal dos trabalhadores o que acaba tornando o cuidado destinado aos pacientes sem interação, formal e rotineiro, mecanizando a assistência.

A realização dessa pesquisa trouxe benefícios para a população em estudo. Possibilitou a reflexão sobre como o estresse pode se manifestar na equipe e influenciar diretamente as relações profissionais e a assistência aos pacientes. O que gerou uma sensibilização desses sujeitos quanto a esse fato, a fim de aprimorarem o seu convívio no setor para trazer melhorias diversas ao ambiente de trabalho e aos doentes por eles assistidos.

Cabe ressaltar que esse estudo foi realizado em um único contexto, caracterizando ações e reflexões de uma população específica, o que gerou aproximações e distanciamentos com a realidade em outras instituições e localidades, sendo necessários novos estudos sobre a temática a fim de ampliar horizontes sobre a relação do estresse e o relacionamento interpessoal da equipe.

\section{REFERÊNCIAS}

1. Silva RM, Beck CCL, Magnago TSBS, Carmagnani MIS, Prestes JPTF. Trabalho noturno e a repercussão na saúde dos enfermeiros. Esc. Anna Nery. [Internet]. 2011;15(2) [acesso em 15 mai 2012]. Disponível: http://www. eean.ufrj.br/revista_enf/vol15jun2/05ARTIGOS/06/ v15n2a6.pdf

2. Assunção RM, Castro PF. Relação entre estresse laboral e personalidade entre profissionais da area de saúde. Rev. Saúde. 2011;5(2):4-16.

3. Rodrigues VMCP, Ferreira ASS. Fatores geradores de estresse em enfermeiros de Unidades de Terapia Intensiva. Rev. Latino-Am. Enfermagem [Internet]. 2011;19(4) [acesso em 14 nov 2013]. Disponível: http:// dx.doi.org/10.1590/S0104-11692011000400023

4. Schmidt DRC, Dantas RAS, Marziale MHP, Laus AM. Estresse ocupacional entre profissionais de enfermagem do bloco cirúrgico. Texto Contexto Enferm. [Internet]. 2009;18(2) [acesso em 14 nov 2013]. Disponível: http:// dx.doi.org/10.1590/S0104-07072009000200017

5. Souza NVDO, Santos DM, Ramos EL, Anunciação CT, Thiengo PCS, Fernandes MC. Repercussões psicofísicas na saúde dos enfermeiros da adaptação e improvisação de materiais hospitalares. Esc. Anna Nery. [Internet] 
2010;14(2) [acesso em 15 mai 2012]. Disponível: http:// dx.doi.org/10.1590/S1414-81452010000200005

6. Casagrande JL, Patrício ZM. Comunidade orgânica no trabalho: Estratégia para a vida saudável do trabalhador e da organização. 1ª ed. Curitiba: CRV; 2010.

7. Ministério da Saúde (BR). Conselho Nacional de Saúde. Resolução № 196, de 10 de outubro de 1996: diretrizes e normas reguladoras de pesquisas envolvendo seres humanos; Brasília: Ministério da Saúde; 1996.

8. Bardin L. Análise de conteúdo. $3^{\mathrm{a}}$ ed. Portugal: Edições 177; 2011.

9. Wagner LR, Thofehrn MB, Amestoy SC, Porto AR, Arrieira ICO. Relações interpessoais no trabalho: percepção de técnicos e auxiliares de enfermagem. Cogitare enferm. [Internet]. 2009;14(1) [acesso em 15 nov 2013]. Disponível: http://ojs.c3sl.ufpr.br/ojs2/index. php/cogitare/article/view/14123/9494

10. Baggio MA. Relações humanas no ambiente de trabalho: o (des)cuidado de si do profissional de enfermagem. Rev. Gaúcha Enferm. [Internet]. 2007;28(3) [acesso em 15 nov 2013]. Disponível: http:// seer.ufrgs.br/RevistaGauchadeEnfermagem/article/ view/4695/2599

11. Cunha AP, Souza EM, Mello R. Os fatores intrínsecos ao ambiente de trabalho como contribuintes da síndrome de burnout em profissionais de enfermagem. R. pesq.: cuid. fundam. [Internet]. 2012;(Suppl.) [acesso em 15 nov 2013]. Disponível: http://www.seer.unirio.br/index. php/cuidadofundamental/article/view/1716/pdf_507

12. Macedo PMC. Desafios atuais no trabalho multiprofissional em saúde. Rev. SBPH [Internet]. 2007;10(2) [acesso em 15 de nov de 2013]. Disponível: http://pepsic. bvsalud.org/pdf/rsbph/v10n2/v10n2a05.pdf

13. Santos $M C$, Bernardes $A$. Comunicação da equipe de enfermagem e a relação com a gerência nas instituições de saúde. Rev. Gaúcha Enferm. [Internet] 2010;31(2) [acesso 13 nov 2013]. Disponível: http:// dx.doi.org/10.1590/S1983-14472010000200022

14. Blanco BM, Valente GSC, Lima CA, Oliveira ACL, Correa MB. A importância da liderança do enfermeiro no gerenciamento das relações interpessoais entre a equipe de saúde. R. pesq.: cuid. fundam. [Internet] 2013;5(3) [acesso em 13 nov 2013]. Disponível: http:// www.seer.unirio.br/index.php/cuidadofundamental/ article/view/1496/pdf_853

15. Oliveira TR, Simões SMF. As faces da comunicação na prática da enfermagem: Uma pesquisa bibliográfica. Rev. bras. enferm. [Internet] 2010; 9(2) [acesso em 19 mai 2012]. Disponível: http://www.objnursing.uff.br/index. php/nursing/article/view/j.1676-4285.2010.3116/698 